\title{
BMJ Open Effectiveness of routine measurement of health-related quality of life in improving the outcomes of patients with musculoskeletal problems - a cluster randomised controlled trial: protocol paper
}

\author{
Cindy Lam, ${ }^{1}$ Weng Yee Chin (D) , ${ }^{1}$ Carlos King Ho Wong (D) , ${ }^{1}$ Kalun Or, ${ }^{2}$ \\ Daniel Yee Tak Fong, ${ }^{3}$ Jason Pui Yin Cheung, ${ }^{4}$ David Vai Kiong Chao, ${ }^{5}$ \\ Eliza L Y Wong (D) , ${ }^{6}$ Paul Kind ${ }^{7,8}$
}

To cite: Lam C, Chin WY, Wong CKH, et al. Effectiveness of routine measurement of health-related quality of life in improving the outcomes of patients with musculoskeletal problems-a cluster randomised controlled trial: protocol paper. BMJ Open 2020;10:e040373. doi:10.1136/ bmjopen-2020-040373

- Prepublication history and additional materials for this paper is available online. To view these files, please visit the journal online (http://dx.doi. org/10.1136/bmjopen-2020040373).

Received 12 May 2020 Revised 19 0ctober 2020 Accepted 08 November 2020

(D) Check for updates

(c) Author(s) (or their employer(s)) 2020. Re-use permitted under CC BY-NC. No commercial re-use. See rights and permissions. Published by BMJ.

For numbered affiliations see end of article.

Correspondence to

Dr Cindy Lam; clklam@hku.hk

\section{ABSTRACT}

Introduction Managing chronic musculoskeletal problems usually focuses on pain control using medications, but outcomes are often unsatisfactory and sometimes harmful. Information on a patient's health-related quality of life (HRQOL) may trigger a doctor to tailor management improving quality of life. The aim of this trial is to find out whether routine measurement and reporting of a patient's EuroQoL 5-Dimension 5-Level (EQ-5D-5L) HRQOL data using an electronic platform can improve HRQOL and pain in patients with chronic knee or back problems in primary care. We will also assess the acceptability of routine electronic measurements and reporting of the EQ-5D-5L in primary care settings.

Methods This is a multicentre, prospective, cluster randomised controlled trial set in six public primary care clinics in Hong Kong. At the intervention clinics, subjects will complete an electronic EQ-5D-5L form at recruitment and at each clinic follow-up over 12 months. A report of the patient's longitudinal EQ-5D-5L data will be provided to the doctor. Subjects in the control clinics will receive care as usual. All subjects will complete the Western Ontario and McMaster Universities 0steoarthritis Index (WOMAC), a 10-point Pain Rating Scale and a structured questionnaire to collect sociodemographic information and data on morbidity and service utilisation at recruitment at baseline, 3, 6 and 12 months. Primary outcome is the change in WOMAC total score. Secondary outcomes are change in pain, other patient-reported outcome scores and doctor-rated severity of disease. Group differences in the changes in WOMAC and other outcome scores over time will be analysed using generalised estimating equation model with an intention-to-treat principle.

Ethics and dissemination Ethics approval has been obtained from The University of Hong Kong/Hospital Authority Hong Kong West Cluster (IRB reference number: UW 18-270). The results of the trial will be submitted for publication in a peer-reviewed journal.

Trial registration number NCT03609762.
Strengths and limitations of this study

This is the first study to evaluate the impact of routine measurements of health-related quality of life (HRQOL) in normal clinical practice in a Chinese population.

- It is an effectiveness-implementation hybrid trial that aims to promote more effective and patientcentred care of people with chronic musculoskeletal problems.

- The study aims to overcome implementation barriers by using modern information technology to collect and generate a patient-filled report on the relevant $\mathrm{HRQOL}$ data with reference to the general population norm at the point of care in real-world primary care clinics.

- If found to be effective, evidence from this study can support the integration of routine HRQOL data collection into electronic medical record systems.

- As the instruments will be collected using electronic tablets, a certain level of computer literacy is required, and the study may not be able to include participants who are unable to perform such tasks which may introduce selection bias associated with age or educational status.

\section{INTRODUCTION}

With ageing of the population and physical inactivity in today's industrialised society, musculoskeletal (MS) problems have become an important disease burden, affecting hundreds of millions of people worldwide. ${ }^{1}$ MS problems account for $7 \%$ of all the diagnoses presenting to primary care in Hong Kong. ${ }^{2}$ Although the spectrum of MS problems is broad, ranging from the common muscle strain, tendinitis and degenerative conditions to the more serious inflammatory joint diseases, they share 
the common characteristics of causing pain and disability. Chronic MS problems are the most common cause of severe chronic pain impairing mental and physical well-being. ${ }^{3}$ The Global Burden of Disease Study 2013 showed that from 2005 to 2013, the major contributor to the global increase of disability-adjusted life years was MS problems. ${ }^{3}$ Four of the top 10 causes of disability in China are MS problems, namely low back pain, neck pain, osteoarthritis and other MS problems. ${ }^{4}$ MS problems also significantly affect the psychosocial health of the patients' families and caregivers. Current clinical management of chronic MS problems focuses predominantly on pain control with medications, but the outcomes are often unsatisfactory. There are increasing concerns regarding serious side effects from the use of non-steroidal anti-inflammatory drugs, and the risk of death and dependency from opioids in the treatment of chronic pain. ${ }^{5}$ More effective interventions are needed to reduce the suffering, disability and service burden arising from MS problems.

The goal of medical care is to improve or restore health and the patient's perspective needs to be taken into account when assessing the outcomes of care. Patientreported outcomes (PRO) are defined as 'any report of the status of a patient's health condition that comes directly from the patient, without interpretation of the patient's response by a clinician or anyone else'. ${ }^{6}$ Pain is a commonly used PRO in clinical practice. Health-related quality of life (HRQOL) is becoming a popular PRO measure in clinical trials. HRQOL is a multidimensional concept that measures a person's subjective perceived effect on how health has affected his/her ability to live a fulfilling life, both physically and mentally. ${ }^{7}$ A variety of HRQOL instruments are available for different purposes, which can be generic or disease specific. ${ }^{7}$ There is a large body of evidence on the psychometrics and clinimetrics of HRQOL measures in assessing the severity of symptoms, ${ }^{8}$ monitoring general health and well-being, ${ }^{9}$ promoting patient-clinician communication, ${ }^{10}$ evaluating treatment/intervention outcomes ${ }^{11-13}$ and informing clinical decision-making. ${ }^{11} 121415$

Up until now, HRQOL has rarely been measured in normal clinical practice because it requires a paradigm shift from objective to subjective outcome measurements, and strategies to overcome the implementation barriers of time, workload, cost and patient burden. ${ }^{12}$

The choice of instrument is critical for successful implementation when measuring HRQOL in normal clinical practice. It should be valid, reliable, applicable to most if not all patients, short and easy to use, and meaningful. The EuroQol-5 Dimension (EQ-5D) questionnaire developed by the EuroQol Group is a generic HRQOL measure that satisfies all these criteria. ${ }^{16}$ It consists of a descriptive system of five HRQOL dimensions (mobility, self-care, usual activities, pain/discomfort and anxiety/ depression) and a $200 \mathrm{~mm}$ visual analogue scale (VAS) for global health rating. It can be completed within a few minutes either by self-completion or by interview administration. The original EQ-5D has three response options to each item (EQ-5D-3L), but was modified to the EuroQoL 5-Dimension 5-Level (EQ-5D-5L) with five response options to each item to improve responsiveness and sensitivity. ${ }^{16}$ The EQ-5D-5L defines a combination of 3125 health states. ${ }^{16}$ Each health state can be converted to a composite utility (preference) score from 0 (death) to 1 (perfect health), with a scoring algorithm derived from population-based valuation. The EQ-5D has been adapted in many countries with population-specific scoring algorithms available in the USA, Canada, Europe and China. A Chinese (Hong Kong) version of the EQ-5D-5L has been validated and the Hong Kong population-specific EQ-5D-5L scoring algorithm has been developed from a population-based valuation study. ${ }^{17} 18$ The EQ-VAS is a $200 \mathrm{~mm}$ vertical scale ranging from 0 to 100 , with 0 corresponding to 'the worst imaginable health' and 100 indicating 'the best imaginable health', and the rating scale scores are an easy-to-interpret composite indicator that can be used to monitor the change in HRQOL over time. The EQ-5D-5L has been shown to be valid, reliable and sensitive in patients with MS problems internationally and in Hong Kong. ${ }^{19}$

\section{Aims and hypothesis}

The aim of this study is to find out whether the implementation of routine measurement and reporting of the patient's EQ-5D-5L HRQOL data with an electronic platform can improve HRQOL and pain in patients with chronic knee or back problems in primary care. We will also assess the acceptability of the implementation of routine electronic measurement and reporting of the EQ-5D-5L HRQOL data in real-world primary care. We hypothesise that the real-time report on longitudinal HRQOL data will facilitate a more patient-centred care with optimisation of the use of drugs, counselling, rehabilitation service, aids and/or surgery to address the patient's physical, social and psychological needs. Furthermore, the EQ-5D report can make the patient more aware of his/her condition, and will become more motivated and empowered to adhere to medical treatments and lifestyle changes where necessary.

\section{METHODS AND ANALYSIS \\ Study design}

This study is a single-blind cluster randomised controlled trial (RCT) to evaluate the effectiveness of measuring and reporting HRQOL in routine clinical practice in improving the outcomes of patients with chronic knee and/or back problems. Randomisation will be performed at the clinic level. Recruited subjects from the control and intervention clinics will be followed up at 3, 6 and 12 months after enrolment into the trial (figure 1).

\section{Subjects}

Recruitment

We will recruit subjects from six primary care clinics, three of which will be randomised as the intervention clinics and three as the control clinics. All adults attending the 


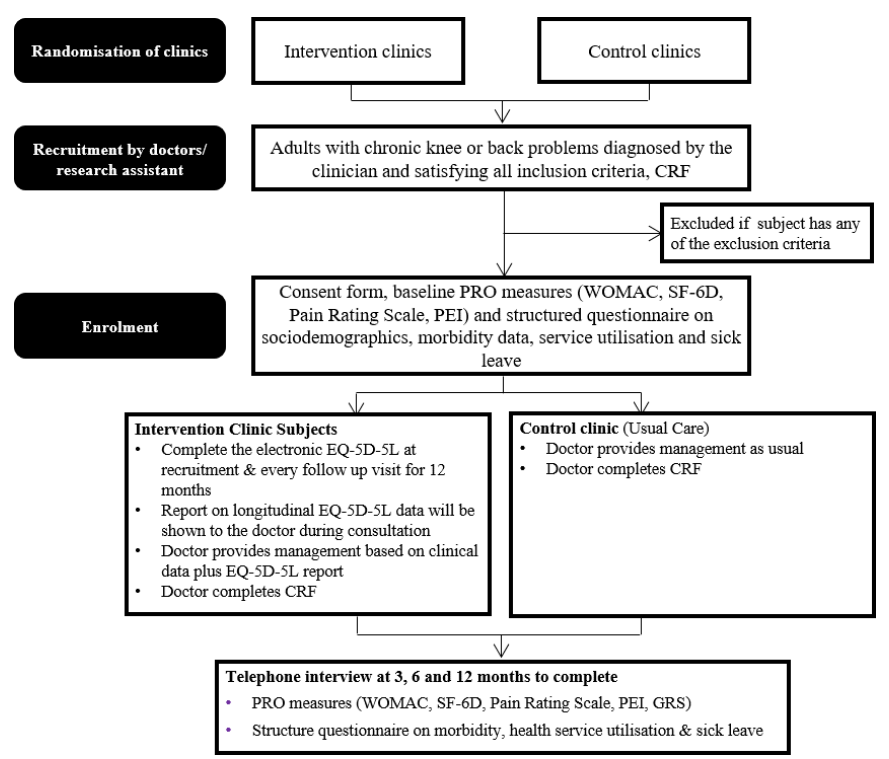

Figure 1 Study flow diagram. CRF, case report form; EQ5D-5L, EuroQoL 5-Dimension 5-Level; GRS, Global Rating Scale; PEI, Patient Enablement Instrument; PRO, patientreported outcome; SF-6D, Short-Form Six-Dimension; WOMAC, Western Ontario and McMaster Universities Osteoarthritis Index.

study clinics for symptomatic chronic knee and/or back pain will be assessed by the attending doctors and invited to join the study if they satisfy the following inclusion criteria and do not meet any of the exclusion criteria.

The inclusion criteria are:

A. Adults aged 18 years or above.

B. Has a doctor-diagnosed symptomatic knee and/or back pain problem that is expected to last for 1 month or more.

C. Scheduled for at least one follow-up visit in the clinic within 12 months.

D. Has given written consent to participate in this study.

The exclusion criteria are:

A. Life expectancy less than 12 months (judged by the doctor).

B. Has cancer. (Patients with current cancers undergoing active or palliative treatment will be excluded since they may have impairment of quality of life from cancer and opioids or other pain relief treatments, which would make an independent evaluation of the outcomes related to the chronic (non-malignant) knee or back pain impossible.)

C. Too ill (both physically or cognitively) to complete a questionnaire.

D. Unable to communicate in Chinese.

E. Does not give consent to participate in the study.

The doctor will complete the clinician-reported baseline clinical case report form (CRF)) for each eligible patient at the end of their consultation. Each subject will sign a written consent form (online supplemental appendix 1) and then complete a structured questionnaire containing items on sociodemographics, service utilisation, pain rating and quality of life.Subjects receuited from the intervention clinics will also complete the electronic EQ-5D-5L on a computer tablet in the clinic after the doctor's consultation. The name and telephone number of each subject will be collected for the follow-up telephone interviews.

To ensure the feasibility and acceptability of the measurement and reporting of the EQ-5D-5L before the doctor consultation in busy outpatient clinics, a pilot study was undertaken in July to December 2018 in two outpatient clinics. All 151 subjects recruited were able to complete the electronic EQ-5D-5L in a mean time of $2 \mathrm{~min}$; $69 \%$ of the patients perceived the electronic EQ-5D-5L survey as both easy to use and understand and $95 \%$ thought the assessment was useful. Among the EQ-5D reports provided to the doctors involved in the pilot, $88 \%$ of the EQ-5D-5L reports were rated as easy to understand and $80 \%$ of the EQ-5D-5L results were rated as being useful by the doctors. The electronic EQ-5D-5L platform operated smoothly with a notebook computer or iPad connected to the clinic public Wi-Fi and the report was able to be generated within seconds.

\section{Randomisation}

A statistician not involved in subject recruitment or data collection will carry out the randomisation of the clinics into the intervention group (three clinics) and control group (three clinics). The allocation will be blinded to the interviewers (assessors) who administer the telephone surveys on the outcomes, and the research team member who will carry out the data analysis.
EQ-5D-5L Utility score

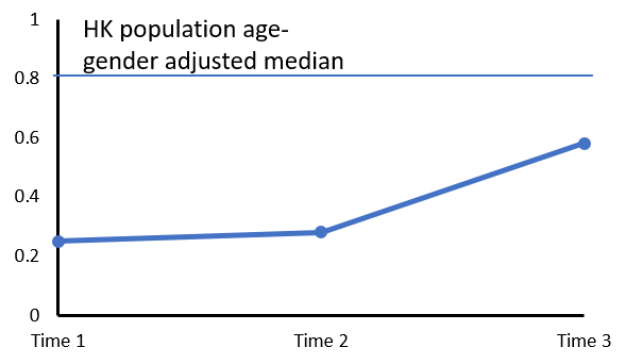

EQ-5D-5L VAS

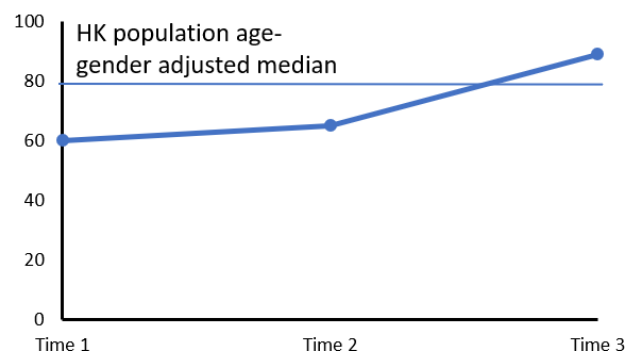

Figure 2 Example of the EuroQoL 5-Dimension 5-Level (EQ-5D-5L) utility score and rating scale score. HK, Hong Kong; VAS, visual analogue scale. 
Table 1 Example of the EuroQoL 5-Dimension 5-Level (EQ5D-5L) data presented to the doctor

\begin{tabular}{|c|c|c|c|c|}
\hline & $\begin{array}{l}\text { HK } \\
\text { population } \\
\text { age-gender } \\
\text { adjusted } \\
\text { median }\end{array}$ & Time 1 & Time 2 & Time 3 \\
\hline Mobility & 1 & 1 & 2 & 1 \\
\hline Self-care & 1 & 1 & 1 & 1 \\
\hline Usual activities & 1 & 1 & 1 & 1 \\
\hline Pain/discomfort & 1 & 2 & 2 & 3 \\
\hline Anxiety/depression & 2 & 2 & 2 & 2 \\
\hline
\end{tabular}

Present the level of each domain at each time point. EQ-5D-5L level: 1=no problems, 2=slight problems, $3=$ moderate problems, 4=severe problems, $5=$ extreme problems. EQ-5D-5L, EuroQoL 5-Dimension 5-Level; HK, Hong Kong.

Intervention group (EQ-5D-5L HRQOL results will be available to the attending doctor)

All subjects attending the intervention clinics will complete the electronic EQ-5D-5L before seeing the doctor during each follow-up visit for the MS problem, a printout of the longitudinal EQ-5D-5L data (table 1) since recruitment will be given to the patients to show to the attending doctors during the consultation. The doctors will provide the management based on the usual clinical information and the additional EQ-5D-5L HRQOL results (figure 2). The doctor will complete the clinician-reported follow-up clinical data form (CRF) of each eligible patient at the end of the consultation.

Control group (usual care, EQ-5D-5L HRQOL results will not be available to the attending doctor)

Subjects attending the control clinics will not need to complete the electronic EQ-5D-5L before seeing the doctor during their follow-up visits. The doctor will manage the patient as usual, based on the usual clinical information. The doctor will complete the clinicianreported follow-up clinical data form (CRF) for each subject at the end of the consultation.

\section{Training and consent of clinicians}

The investigators (CL, JPYC, CKHW) will provide a training session on the interpretation of the EQ-5D-5L HRQOL profile and VAS scores to all doctors of the intervention clinics. Each participating doctor will complete a consent form (online supplemental appendix 2) to take part in the study.

\section{Outcome measures}

Primary outcome

The primary outcome is the change in HRQOL measured by the Western Ontario and McMaster Universities Osteoarthritis Index (WOMAC) total score.
Secondary outcomes

1. Change in pain score as measured by the 10-point Pain Scale.

2. Change in PROs: Short-Form Six-Dimension (SF-6D) utility score, global health measured by the Global Rating Scale (GRS), patient enablement measured by the Patient Enablement Instrument (PEI) score.

3. Change in clinician-reported disease severity and progression.

\section{Exploratory outcomes}

1. Health service utilisation rates.

2. Drug and other treatment utilisation rates.

3. Patient acceptability of routine measurement of HRQOL by the electronic EQ-5D-5L.

\section{Confounders}

Clinical settings, sociodemographics, MS diagnosis, duration and severity, treatment and comorbidity.

\section{Sample size calculation}

The sample size calculation was based on the primary outcome of a difference in WOMAC total score between the intervention and control groups. A previous RCT study evaluating a rehabilitation programme integrating exercise, self-management and active coping strategies for patients with chronic knee pain showed that the average change in WOMAC total score at 6 months after baseline for the intervention and control groups was -3.4 (mean: 35; SD: 16 at 6 months) and -8 (mean: 30.4; SD: 17 at 6 months), respectively, ${ }^{20}$ indicating an effect size of 0.3. Using this, a minimum of 380 (190 in each group) patients are needed to achieve $80 \%$ power at $5 \%$ significant level, by two-sample t-test. There were no studies that provided an intracluster correlation (ICC) coefficient for WOMAC scores, but a review showed the ICC coefficient of pain outcome was $0.03^{21}$; to adjust for the clustering effect from six clinics, a larger sample size of 1098 (549 patients per group, and 183 subjects per clinic) will be required. We plan to recruit 1374 (687 in each group) subjects to allow for a $20 \%$ attrition rate.

\section{Study instruments}

Chinese (Hong Kong) EQ-5D-5L and the acceptability of routine completion of the electronic EQ-5D-5L:

1. The electronic Chinese EQ-5D-5L comprises a descriptive system of five questions on five HRQOL dimensions (mobility, self-care, usual activities, pain/ discomfort and anxiety/depression) and a $100 \mathrm{~mm}$ (adapted from $200 \mathrm{~mm}$ to fit the Tablet screen) VAS on global health from 0 corresponding to 'the worst imaginable health' to 100 indicating 'the best imaginable health'. Each question has five response options ( $1=$ no problems, $2=$ slight problems, $3=$ moderate problems, $4=$ severe problems, $5=$ extreme problems). The responses to the five questions give an HRQOL profile. ${ }^{16}$

2. The acceptability of routine completion of the electronic EQ-5D-5L will be evaluated by the perceived ease of use and perceived usefulness scales based on 
the technology acceptability model, which was translated and validated on a local Chinese population. ${ }^{22}$ Their Cronbach's alpha was 0.90 and 0.89 , respectively. They had good convergent validity with significant correlation of 0.69 .

PROs on:

1. Chinese Western Ontario and McMaster Universities Osteoarthritis Index (WOMAC): the WOMAC is a widely used condition-specific HRQOL measure to assess pain, stiffness and physical function $(\mathrm{PF})$ of patients with MS problems. It has been applied to patients with hip and/or knee osteoarthritis, low back pain, rheumatoid arthritis and fibromyalgia. ${ }^{23}$ It consists of 24 items on three domains: pain (5 items), stiffness (2 items) and PF (17 items). Each question is rated on a 5-point Likert scale from 0 to 4, with lower scores indicating lower levels of symptoms or impairment. The item scores in each domain subscale are summated to a domain score. The total WOMAC score is calculated by summating the three domain scores. A Chinese version of the WOMAC is available and has been shown to be valid, reliable and sensitive in Chinese patients. ${ }^{24}$

2. Ten-point Pain Scale: the patient will be asked to rate their pain that best represents the intensity of their pain using a scale from 0 to 10 , where 0 indicates no pain and 10 indicates the worst pain.

3. Chinese Short-Form Six-Dimension (SF-6D): the SF-6D is a preference-based measure of health, derived from the 36-Item Short Form Health Survey, that generates a utility score. It has six items measuring six HRQOL dimensions of physical functioning, role limitation, social functioning, bodily pain, mental health and vitality. The SF-6D responses have a combination of 18000 health states. Each health state can be converted to a composite preference (utility) score from 0 indicating death to 1 indicating perfect health. The Chinese SF$6 \mathrm{D}$ was validated in the Chinese population in Hong Kong, ${ }^{25}$ and a Hong Kong population-based scoring algorithm has been established. The mean SF-6D utility score of the Hong Kong Chinese population is $0.78 .^{25}$

4. Chinese Patient Enablement Index (PEI): the PEI is a six-item questionnaire that measures the patient's perceived change in coping with illness and self-care. Each item is rated on a 3-point (0,1 and 2) scale. The sum of the item scores gives the final PEI score, with higher scores indicating better enablement. The Chinese version was shown to be a valid, reliable and sensitive outcome measure among Chinese patients in Hong Kong. ${ }^{26}$

5. Global Rating Scale (GRS): the GRS on change in health will be used to assess the patient's self-perception of any change in the overall health condition on a 7-point scale: -3 (much worse), -2 (worse), -1 (a little worse), 0 (no change), 1 (a little better), 2 (better), 3 (much better). ${ }^{27}$

A structured questionnaire (online supplemental appendix 3-baseline and follow-up) on
1. Sociodemographic characteristics including age, sex, education background, marital status, occupation, monthly household income, smoking and drinking status.

2. Comorbidities: the presence of doctor-diagnosed chronic diseases including hypertension, diabetes, stroke, heart disease, pulmonary diseases including asthma and chronic obstructive pulmonary disease, mental illness, renal disease, osteoporosis, cancer and others.

3. Health service utilisation: monthly frequency of outpatient consultations with Western/Chinese medicine practitioners, allied health service visits, accident and emergency department attendance and admissions to hospitals.

4. Days of sick leave in the past 1 month.

5. Medication use in the past 1 month.

Clinician-reported clinical case report form (CRF) (online supplemental appendix 4-baseline and follow-up) on:

1. Disease diagnosis and duration: the diagnosis and duration of the chief MS problem will be reported by the attending doctor.

2. Global rating on disease severity: the attending doctor will rate the patient's disease severity on a 5-point Likert scale: $1=$ no problem, $2=$ mild, $3=$ moderate, $4=$ severe, $5=$ very severe.

3. Global rating on disease progression: in each followup clinic consultation, the attending doctor will rate the patient's disease progression on a 7-point scale: -3 (much worse), -2 (worse), -1 (a little worse), 0 (no change), 1 (a little better), 2 (better), 3 (much better).

4. Disease management: the management modalities including investigations, drugs (type and dosage), referral to allied health service (types and frequency) and surgery (type and year of the surgery) will be extracted from the medical record.

5. Perceived usefulness of the EQ-5D-5L data in enhancing patient management.

\section{Data collection}

A computer program will be developed to administer the electronic version of the Chinese (Hong Kong) EQ-5D-5L on a computer tablet, to calculate the utility score and to generate a report on the longitudinal EQ-5D-5L profile scores, preference scores and VAS scores in a printable form (table 1 and figure 2).

All subjects from the intervention clinics will complete the electronic Chinese (Hong Kong) EQ-5D-5L at recruitment and during every follow-up visit at the clinic before the doctor consultation over the next 12 months.

To control for potential confusing variables and errors, a trained research assistant will assist the subjects in the completion of the electronic EQ-5D-5L. All other patient data will be collected by interviewers who will be trained by the project coordinator to ensure all questions are asked in a standardised manner. We will conduct briefing 
meetings with the doctors in charge of all participating clinics and send a written briefing of the study and a guide on the interpretation of the EQ-5D-5L report to each participating doctor.

The doctor will complete the CRF (online supplemental appendix 4) for each study subject after the consultation at recruitment and during every follow-up consultation over the next 12 months for subjects from both the intervention and control clinics.

A survey on sociodemographic, comorbidity, PRO and health service utilisation will be administered to each subject within 2 weeks from recruitment (baseline), and the survey on PRO and health service utilisation will be repeated by telephone at 3,6 and 12 months (blinded to group allocation) after the baseline survey. The telephone survey will be carried out by the HKU Social Science Research Centre. Data collection at baseline and follow-up for intervention and control groups is shown in table 2 .

\section{Data processing and analysis}

Data will be analysed by intention to treat, in which all subjects who are randomised and have completed at least one repeat outcome measurement will be included in the analysis. Multiple imputations will be used to substitute missing data. Complete case analysis will also be conducted to confirm the results.

The within-group changes in WOMAC, pain and SF-6D scores will be compared by paired t-test, or Wilcoxon signed-rank test if the outcome data are not normally distributed. Between-group difference of changes in WOMAC, pain and SF-6D scores, and between-group difference in GRS and PEI scores at the end of 12 months will be compared by two-sample t-test, or Mann-Whitney $\mathrm{U}$ test if the outcome data are not normally distributed.

The differences between groups in outcomes, including WOMAC, pain, SF-6D, GRS and PEI, will be assessed by the generalised estimating equations (GEE).$^{28}$ Specifically, we shall use the identity link to regression on group, baseline value and time, and allow extra-covariance of repeated from the same subject and different subjects from the same cluster by an unstructured working covariance matrix. Between-group differences in clinician-rated disease severity rates of medication use, service utilisation and sick leave will be similarly assessed by GEE but with a Poisson link to cater the different measurement scale of the outcomes. The acceptability of measurement of routine HRQOL measurement by the electronic EQ-5D-5L will be summarised by descriptive statistics on patient and doctor evaluation.

The STATA software V.13 (StataCorp, Texas) will be used for the data analysis. A $5 \%$ level of significance will be used in all significance tests.

\section{Data monitoring}

An external data monitoring committee was not deemed to be necessary for this trial. Data will be monitored by
Table 2 Data collection at baseline and follow-up for intervention and control groups

\begin{tabular}{|c|c|c|c|c|}
\hline & Baseline & & Follow-up & \\
\hline & Intervention & Control & Intervention & Control \\
\hline \multicolumn{5}{|c|}{ 1. Patient-reported outcomes } \\
\hline$E Q-5 D-5 L$ & $x$ & $x$ & $x$ & $x$ \\
\hline WOMAC & $x$ & $x$ & $x$ & $x$ \\
\hline SF-6D & $x$ & $x$ & $x$ & $x$ \\
\hline PEI & & & $x$ & $x$ \\
\hline GRS & & & $x$ & $x$ \\
\hline $\begin{array}{l}\text { Patient acceptability } \\
\text { questionnaire }\end{array}$ & $x$ & $x$ & & \\
\hline \multicolumn{5}{|c|}{ 2. Sociodemographic status and lifestyle } \\
\hline Age & $\times$ & $x$ & & \\
\hline Sex & $x$ & $x$ & & \\
\hline $\begin{array}{l}\text { Education } \\
\text { background }\end{array}$ & $x$ & $x$ & & \\
\hline Occupation & $x$ & $x$ & & \\
\hline $\begin{array}{l}\text { Monthly household } \\
\text { income }\end{array}$ & $x$ & $x$ & & \\
\hline Smoking & $x$ & $x$ & & \\
\hline Drinking & $x$ & $x$ & & \\
\hline \multicolumn{5}{|l|}{ 3. Burden of illness } \\
\hline $\begin{array}{l}\text { Medical service } \\
\text { utilisation }\end{array}$ & $x$ & $x$ & $x$ & $x$ \\
\hline Sick leave & $x$ & $x$ & $x$ & $x$ \\
\hline Use of medication & $x$ & $x$ & $\times$ & $\times$ \\
\hline \multicolumn{5}{|c|}{ 4. Clinician-reported outcomes } \\
\hline $\begin{array}{l}\text { Global rating on } \\
\text { disease severity }\end{array}$ & $x$ & $x$ & $x$ & $x$ \\
\hline $\begin{array}{l}\text { Global rating on } \\
\text { disease progression }\end{array}$ & & & $x$ & $x$ \\
\hline $\begin{array}{l}\text { Doctor acceptability } \\
\text { questionnaire }\end{array}$ & & & $x$ & $x$ \\
\hline \multicolumn{5}{|l|}{ 5. Clinical data } \\
\hline $\begin{array}{l}\text { Disease diagnosis } \\
\text { and duration }\end{array}$ & $x$ & $x$ & & \\
\hline Drug treatment & $x$ & $x$ & $x$ & $x$ \\
\hline Comorbidities & $x$ & $x$ & $x$ & $x$ \\
\hline $\begin{array}{l}\text { Investigations/ } \\
\text { referrals }\end{array}$ & $x$ & $x$ & $x$ & $x$ \\
\hline
\end{tabular}

EQ-5D-5L, EuroQoL 5-Dimension 5-Level; GRS, Global Rating Scale; PEI, Patient Enablement Instrument; SF-6D, Short-Form SixDimension; WOMAC, Western Ontario and McMaster Universities Osteoarthritis Index.

the research team which includes clinicians, statisticians and information technology experts. This study is considered to be a low-risk trial where both the intervention and control groups will receive their usual medical care. Rules for early stopping have not been implemented as the clinicians involved in this study are not blinded to intervention allocation. 
Collection and assessment of reported adverse events and other unintended effects of the trial interventions or trial conduct will be performed on a continuous basis as such events arise. Queries identified will be resolved promptly by the research team. All unintended effects and adverse events will be reported every 6 months to the Institutional Review Board (IRB) of the University of Hong Kong and Queen Mary Hospital. Interim analyses will be reported to the IRB and funding body every 6 months. The principal author (CL) will be responsible for overseeing the interim analyses and any decisions to stop the trial.

\section{Patient and public involvement}

This research is planned to be done without patient involvement. Patients will not be invited to comment on the study design and will not be consulted to develop patient-relevant outcomes or interpret the results. Patients will not be invited to contribute to the writing or editing of the future manuscript for readability or accuracy.

\section{DISCUSSION}

Routine measurement of HRQOL in normal clinical practice has been used in selected patient populations, mostly in oncology ${ }^{29}$ and specific surgical settings in the UK and Sweden. ${ }^{14}$ In the UK, as a government initiative to compare service providers' performance, HRQOL has been routinely measured by the EQ-5D before and after the National Health Service funded elective hip or knee replacement, varicose vein and inguinal hernia surgeries since 2009. This changes the

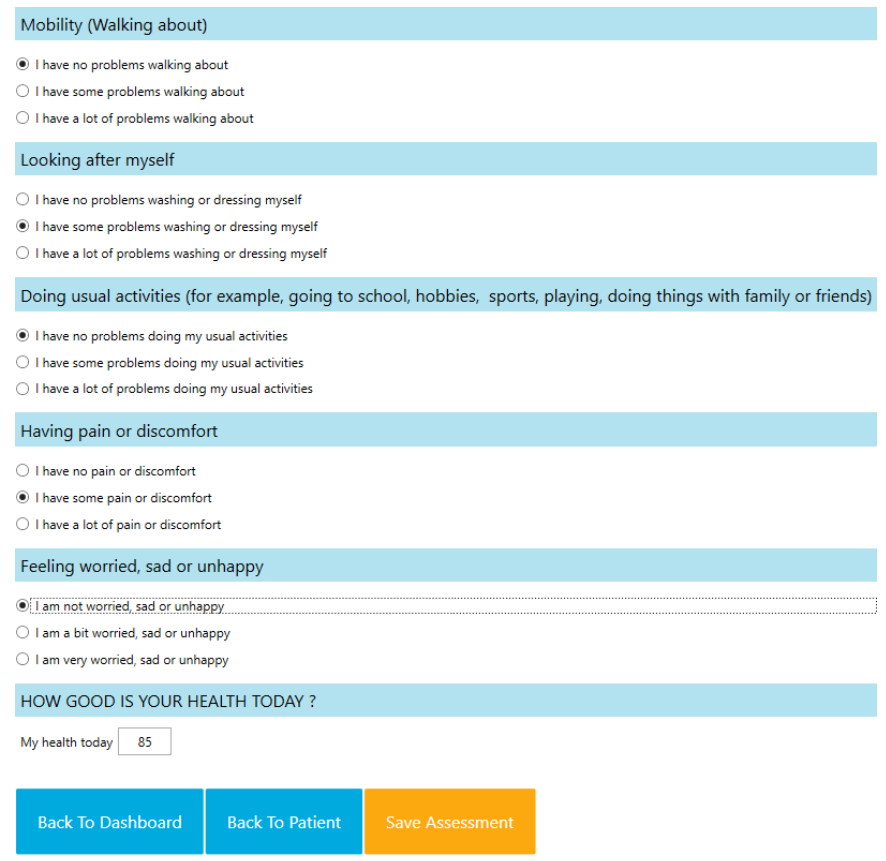

Figure 3 Example of an electronic platform for measuring and scoring EuroQol-5 Dimension (EQ-5D). paradigm of evaluation of effectiveness of care from the clinician to the patient's perspective in order to improve accountability. ${ }^{29}$ It also enables patients to make an informed choice on service providers. ${ }^{29}$ In Sweden, the Swedish Hip Arthroplasty Register has recorded the EQ-5D utility score and a VAS pain score of each patient since 2002, and demonstrated that the overall PRO of total hip replacement surgery is satisfactory, leading to a mean increase of 0.36 in EQ-5D utility score. ${ }^{14}$ Data on routine measurement of HRQOL by the EQ-5D or other measures in busy outpatient settings are currently lacking, and we could not find any study on routine measurement of HRQOL in normal clinical practice in Chinese populations.

In preparation for this study, we have developed an electronic platform for the administration, scoring and reporting of EQ-5D-5L data in normal outpatient clinics, which can make the results available to the attending doctors in real time (a mock example is shown in figure 3 ). If found to be beneficial, routine measurement of HRQOL by an electronic EQ-5D-5L platform may be applied to other outpatient clinics to help improve the care of MS problems and other conditions, and to facilitate more effective and patient-centred care.

\section{DATA MANAGEMENT AND OVERSIGHT}

Members of the research team from the University of Hong Kong will take responsibility for the conduct of research staff and study participants to ensure protocol compliance, proper study management and timely completion of study procedures. The Consolidated Standards of Reporting Trials table with the RCT plan is available at online supplemental appendix 5 .

\section{PROTOCOL AND REGISTRATION}

The trial is registered with the US Clinical Trial Registration at ClinicalTrials.gov; protocol ID HKUCTR-2418.

\section{DATA STORAGE SECURITY AND PATIENT CONFIDENTIALITY}

Personal identifiable information will be deidentified and replaced with research identification codes. Data sets will have all personal identifiers removed so that participant identities cannot be determined in case of data security breaches. Cover and consent sheets containing identifiable information will be stored separately from any completed survey instruments and access to master code lists will be limited to the investigation team only. Individual files containing electronic data will be password protected and encrypted and stored on institutional network drives with firewalls and security measures in place. Consent forms and other hard copy records will be stored securely 
in a locked cabinet in a secure location separate from where the research data are kept. All research staff will be trained in the IRB-approved methods for managing and storing research data.

\section{ETHICS AND DISSEMINATION}

Ethics approval hasbeen obtained from Institutional Review Board of The University of Hong Kong/Hong Kong/ Hospital Authority Hong Kong West Cluster (IRB Reference Number: UW18-270) who have reviewed and approved the study procedures, ethics, subjectinformation and consent, and subject safety. The results of the trial will be submitted for publication in a peer-reviewed journal.

\section{Author affiliations}

${ }^{1}$ Department of Family Medicine and Primary Care, University of Hong Kong Li Ka

Shing Faculty of Medicine, Hong Kong, China

${ }^{2}$ Industrial and Manufacturing Systems Engineering, University of Hong Kong

Faculty of Engineering, Hong Kong, China

${ }^{3}$ School of Nursing, University of Hong Kong Li Ka Shing Faculty of Medicine, Hong Kong, China

${ }^{4}$ Department of Orthopaedics and Traumatology, University of Hong Kong Li Ka Shing Faculty of Medicine, Hong Kong, China

${ }^{5}$ Department of Family Medicine and Primary Health Care, United Christian Hospital, Hong Kong, China

${ }^{6} \mathrm{JC}$ School of Public Health and Primary Care, The Chinese University of Hong Kong, Hong Kong, China

${ }^{7}$ Institute of Health Sciences, University of Leeds, Leeds, UK

${ }^{8}$ Centre for Health Economics, Management and Policy, National Research

University Higher School of Economics, Moskva, Russia

Contributors CL conceptualised the study and obtained the funding. CL, WYC, CKHW, DYTF, KO, JPYC and ELYW contributed to the development of study design. DYTF, KO, JPYC and DVKC were responsible for the development of the data collection platform, field testing of the study logistics, including clinic and subject recruitment. CL, CKHW, DYTF and PK were responsible for the analysis and interpretation of results. CL, CKHW and WYC drafted the first version of the manuscript. All authors read, edited and approved the final version.

Funding This study was funded by the Research Grant Council-General Research Fund, Hong Kong (RGC reference number 17100119).

Disclaimer The funding organisation has not played any role in the design and conduct of the study; collection, management, analysis, or interpretation of the data; or preparation of the manuscript.

Competing interests None declared.

Patient consent for publication Not required.

Provenance and peer review Not commissioned; externally peer reviewed.

Supplemental material This content has been supplied by the author(s). It has not been vetted by BMJ Publishing Group Limited (BMJ) and may not have been peer-reviewed. Any opinions or recommendations discussed are solely those of the author(s) and are not endorsed by BMJ. BMJ disclaims all liability and responsibility arising from any reliance placed on the content. Where the content includes any translated material, BMJ does not warrant the accuracy and reliability of the translations (including but not limited to local regulations, clinical guidelines, terminology, drug names and drug dosages), and is not responsible for any error and/or omissions arising from translation and adaptation or otherwise.

Open access This is an open access article distributed in accordance with the Creative Commons Attribution Non Commercial (CC BY-NC 4.0) license, which permits others to distribute, remix, adapt, build upon this work non-commercially, and license their derivative works on different terms, provided the original work is properly cited, appropriate credit is given, any changes made indicated, and the use is non-commercial. See: http://creativecommons.org/licenses/by-nc/4.0/.
ORCID iDs

Weng Yee Chin http://orcid.org/0000-0003-3171-6792

Carlos King Ho Wong http://orcid.org/0000-0002-6895-6071

Eliza L Y Wong http://orcid.org/0000-0001-9983-6219

\section{REFERENCES}

1 Smith E, Hoy DG, Cross M, et al. The global burden of other musculoskeletal disorders: estimates from the global burden of disease 2010 study. Ann Rheum Dis 2014;73:1462-9.

2 Lo Y, Lam C, Lam T, et al. Hong Kong primary care morbidity survey 2007-2008. Hong Kong Practitioner 2010;32:17-26.

3 Murray CJL, Barber RM, et al, GBD 2013 DALYs and HALE Collaborators. Global, regional, and national disability-adjusted life years (DALYs) for 306 diseases and injuries and healthy life expectancy (HALE) for 188 countries, 1990-2013: quantifying the epidemiological transition. Lancet 2015;386:2145-91.

4 Yang G, Wang Y, Zeng Y, et al. Rapid health transition in China, 19902010: findings from the global burden of disease study 2010. Lancet 2013;381:1987-2015.

5 Gomes T, Juurlink DN, Antoniou T, et al. Gabapentin, opioids, and the risk of opioid-related death: a population-based nested case-control study. PLoS Med 2017;14:e1002396.

6 Speight J, Barendse SM. Fda guidance on patient reported outcomes. BMJ 2010;340:c2921.

7 Guyatt GH, Feeny DH, Patrick DL. Measuring health-related quality of life. Ann Intern Med 1993;118:622-9.

8 Levine DW, Simmons BP, Koris MJ, et al. A self-administered questionnaire for the assessment of severity of symptoms and functional status in carpal tunnel syndrome. J Bone Joint Surg Am 1993;75:1585-92.

9 Glaser AW, Fraser LK, Corner J, et al. Patient-reported outcomes of cancer survivors in England 1-5 years after diagnosis: a cross-sectional survey. BMJ Open 2013;3 doi:10.1136/ bmjopen-2012-002317

10 Berry DL, Blumenstein BA, Halpenny B, et al. Enhancing patientprovider communication with the electronic self-report assessment for cancer: a randomized trial. $J$ Clin Oncol 2011;29:1029-35.

11 Black N. Patient reported outcome measures could help transform healthcare. BMJ 2013;346:f167.

12 Basch E, Abernethy AP. Supporting clinical practice decisions with real-time patient-reported outcomes. J Clin Oncol 2011;29:954-6.

13 Rolfson O, Kärrholm J, Dahlberg LE, et al. Patient-reported outcomes in the Swedish hip arthroplasty register: results of a nationwide prospective observational study. J Bone Joint Surg $\mathrm{Br}$ 2011;93:867-75

14 Lavallee DC, Chenok KE, Love RM, et al. Incorporating patientreported outcomes into health care to engage patients and enhance care. Health Aff 2016;35:575-82.

15 Nicolaije KAH, Ezendam NPM, Vos MC, et al. Impact of an automatically generated cancer survivorship care plan on patientreported outcomes in routine clinical practice: Iongitudinal outcomes of a pragmatic, cluster randomized trial. $J$ Clin Oncol 2015;33:3550-9.

16 Herdman M, Gudex C, Lloyd A, et al. Development and preliminary testing of the new five-level version of EQ-5D (EQ-5D-5L). Qual Life Res 2011;20:1727-36.

17 Wong EL, Yeoh EK, Slaap B, et al. Validation and valuation of the Preference-Based Healthindex using Eq-5d-5l in the Hong Kong population. Value Health 2019;22(8):916-24.

18 Wong ELY, Ramos-Goñi JM, Cheung AWL, et al. Assessing the use of a feedback module to model EQ-5D-5L health states values in Hong Kong. Patient 2018;11:235-47.

19 Cheung PWH, Wong CKH, Samartzis D, et al. Psychometric validation of the EuroQoL 5-Dimension 5-Level (EQ-5D-5L) in Chinese patients with adolescent idiopathic scoliosis. Scoliosis Spinal Disord 2016;11:19.

20 Hurley MV, Walsh NE, Mitchell HL, et al. Clinical effectiveness of a rehabilitation program integrating exercise, self-management, and active coping strategies for chronic knee pain: a cluster randomized trial. Arthritis Rheum 2007;57:1211-9.

21 Campbell M, Grimshaw J, Steen N. Sample size calculations for cluster randomised trials. changing professional practice in Europe group (EU Biomed II concerted action). J Health Serv Res Policy 2000;5:12.

22 Yan M, Or C. A 12-week pilot study of acceptance of a computerbased chronic disease self-monitoring system among patients with 
type 2 diabetes mellitus and/or hypertension. Health Informatics $J$ 2017;1460458217724580.

23 Bellamy N, Buchanan WW, Goldsmith $\mathrm{CH}$, et al. Validation study of WOMAC: a health status instrument for measuring clinically important patient relevant outcomes to antirheumatic drug therapy in patients with osteoarthritis of the hip or knee. $J$ Rheumatol 1988;15:1833-40.

24 Symonds T, Hughes B, Liao S, et al. Validation of the Chinese Western Ontario and McMaster universities osteoarthritis index in patients from mainland China with osteoarthritis of the knee. Arthritis Care Res 2015;67:1553-60.

25 Lam CLK, Brazier J, McGhee SM. Valuation of the SF-6D health states is feasible, acceptable, reliable, and valid in a Chinese population. Value Health 2008;11:295-303.
26 Lam CLK, Yuen NYK, Mercer SW, et al. A pilot study on the validity and reliability of the patient enablement instrument (PEI) in a Chinese population. Fam Pract 2010;27:395-403.

27 Jaeschke R, Singer J, Guyatt GH. Measurement of health status. ascertaining the minimal clinically important difference. Control Clin Trials 1989;10:407-15.

28 Higgins J, Eldridge S, Li T. Chapter 23: including variants on randomized trials: cochrane training. Available: https://training. cochrane.org/handbook/current/chapter-23\#section-23-1-3 [Accessed 8 Aug 2020].

29 National Health Service. Patient reported outcome measures (PROMs). Available: http://content.digital.nhs.uk/proms-background [Accessed 5 Sep 2017]. 